\title{
Long-Distance Spin-Spin Coupling via Floating Gates
}

\author{
Luka Trifunovic, ${ }^{1}$ Oliver Dial, ${ }^{2}$ Mircea Trif, ${ }^{1,3}$ James R. Wootton, ${ }^{1}$ Rediet Abebe, ${ }^{2}$ Amir Yacoby, ${ }^{2}$ and Daniel Loss ${ }^{1}$ \\ ${ }^{1}$ Department of Physics, University of Basel, Klingelbergstrasse 82, CH-4056 Basel, Switzerland \\ ${ }^{2}$ Department of Physics, Harvard University, Cambridge, Massachusetts 02138, USA \\ ${ }^{3}$ Department of Physics and Astronomy, University of California, Los Angeles, California 90095, USA \\ (Received 26 October 2011; published 26 January 2012)
}

\begin{abstract}
The electron spin is a natural two-level system that allows a qubit to be encoded. When localized in a gate-defined quantum dot, the electron spin provides a promising platform for a future functional quantum computer. The essential ingredient of any quantum computer is entanglement-for the case of electronspin qubits considered here-commonly achieved via the exchange interaction. Nevertheless, there is an immense challenge as to how to scale the system up to include many qubits. In this paper, we propose a novel architecture of a large-scale quantum computer based on a realization of long-distance quantum gates between electron spins localized in quantum dots. The crucial ingredients of such a long-distance coupling are floating metallic gates that mediate electrostatic coupling over large distances. We show, both analytically and numerically, that distant electron spins in an array of quantum dots can be coupled selectively, with coupling strengths that are larger than the electron-spin decay and with switching times on the order of nanoseconds.
\end{abstract}

DOI: 10.1103/PhysRevX.2.011006

Subject Areas: Nanophysics, Quantum Information, Semiconductor Physics

\section{INTRODUCTION}

Spins of electrons confined to quantum dots provide one of the most promising platforms for the implementation of a quantum computer in solid-state systems. The last decade has seen steady and remarkable experimental progress in the quantum control and manipulation of single spins in such nanostructures on very fast time scales down [1] to $200 \mathrm{ps}$ and with coherence times of $270 \mu \mathrm{s}$ [2].

A large-scale quantum computer must be capable of reaching a system size of thousands of qubits, in particular, to accommodate the overhead for quantum error correction [3]. This requirement poses serious architectural challenges for the exchange-based quantum-dot scheme [4], since-with present-day technology-there is hardly enough space in which to place the large amount of metallic gates and wires needed to define and address the spin qubits. A promising strategy to meet this challenge is to implement long-range interactions between the qubits. These interactions allow the quantum dots to be moved apart and create space for the wirings. Based on such a design, we propose a quantum-computer architecture that consists of a two-dimensional lattice of spin qubits, with nearest-neighbor (and beyond) qubit-qubit interaction. Such an architecture provides a platform for implementing the surface code-the most powerful fault-tolerant quantum-error-correction scheme known, with an exceptionally large error threshold of $1.1 \%[5,6]$.

Published by the American Physical Society under the terms of the Creative Commons Attribution 3.0 License. Further distribution of this work must maintain attribution to the author(s) and the published article's title, journal citation, and DOI.
To achieve such long-range interactions, we propose a mechanism for entangling spin qubits in quantum dots (QDs) based on floating gates and spin-orbit interaction. The actual system we analyze is composed of two double QDs which are not tunnel coupled. The number of electrons in each double QD can be controlled efficiently by tuning the potential on nearby gates. Moreover, the electrons can be moved from the left to the right dot within each double QD by applying strong bias voltage. Thus, full control over the double QD is possible by only electrical means. The double QDs are separated by a large distance compared to their own size so that they can interact only capacitively. An electromagnetic cavity $[7,8]$ can be used to create a long-range qubit-qubit coupling $[9,10]$. In this paper, we consider the classical limit of this coupling, i.e., a metallic floating gate [11-14] suspended over the two double QDs, or a shared two-dimensional electron-gas (2DEG) lead between the qubits. The strength of the coupling mediated by this gate depends on its geometry, as well as on the position and orientation of the double QDs underneath the gate. Finally, we show that spin qubits based on spins $-\frac{1}{2}$ [4] and on singlet-triplet states [15] can be coupled, and thus hybrid systems can be formed that combine the advantages of both spin-qubit types.

\section{ELECTROSTATICS OF THE FLOATING GATE}

The Coulomb interaction and spin-orbit interaction (SOI) enable coupling between spin qubits of different QD systems in the complete absence of tunneling [16-19]. However, the Coulomb interaction is screened at large distances by electrons of the 2DEG and of the metal gates. Thus, the long-distance coupling between two spin qubits is not feasible via direct Coulomb interaction. However, by 
exploiting long-range electrostatic forces, it has been demonstrated experimentally $[11,12]$ that QDs can be coupled and controlled capacitively via floating metallic gates over long distances. The optimal geometric design of such floating gates should be such that the induced charge stays as close as possible to the nearest QDs and does not spread out uniformly over the entire gate surface. In other words, the dominant contributions to the total gate capacitance should come from the gate regions that are near the QDs. To achieve a strong qubit-qubit coupling, there is one more requirement: The electric field induced on one QD needs to be sensitive to the changes of the charge distribution of the other QD. Thus, the charge gradient, $\left(\partial q_{\text {ind }} / \partial \boldsymbol{r}\right)_{\mid r=0}$, needs to be large, where $r$ is the position vector of the point charge with respect to the center of the respective QD. To fulfill these requirements, we assume that the floating gates consist of two metallic discs of radius $R$ joined by a thin wire of length $L$.

Let us now investigate the optimal design by modeling the electrostatics of the floating gates. The electron charge in the QD induces an image charge of opposite sign on the nearby disc (ellipsoid). See Fig. 1. Because the gate voltage is floating with respect to the ground, the excess charge is predominantly distributed on the distant metallic ellipsoid, thus producing an electric field that acts on the second QD. To carry out the quantitative analysis of the electrostatic coupling, we make use of the expression for an induced charge on the grounded ellipsoidal conductor in the field of a point charge [20]. Electrostatic considerations imply that the coupling (gradient) is enhanced by implementing a flatdisc design of the gate. Thus, in what follows, we set the disc height to zero. To reach this regime in practice, one only has to ensure that the disc height be much smaller than its radius. The expression for the induced charge (in the units of the electron charge) is then given by [20]

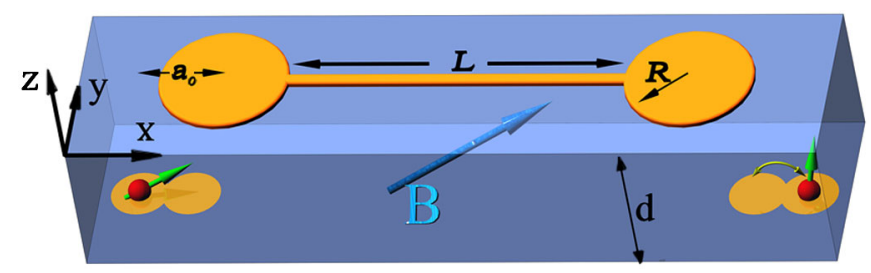

FIG. 1. Model system consisting of two identical double QDs (paired small orange discs) in the $x-y$ plane and the floating gate between them. The gate consists of two metallic discs of radius $R$ connected by a thin wire of length $L$. Each double QD can accommodate one or two electrons (red spheres), defining the corresponding qubit. Absence of tunneling between the separate double QD is assumed; the purely electrostatic interaction between the electrons in the double QDs leads to an effective qubit-qubit coupling. For the spin- $-\frac{1}{2}$ qubit, the coupling depends sensitively on the orientation of the magnetic field $B$. Here, $a_{0}$ is the in-plane distance between a QD and the corresponding disc's center, while $d$ is the vertical distance between the QD and the gate.

$$
q_{\text {ind }}(\boldsymbol{r})=\frac{2}{\pi} \arcsin \left(R / \xi_{r}\right),
$$

where $R$ is the radius of the disc. The ellipsoidal coordinate $\xi_{r}$ is given by

$$
\begin{aligned}
2 \xi_{\boldsymbol{r}}^{2}= & R^{2}+d^{2}+\left|\boldsymbol{a}_{0}+\boldsymbol{r}\right|^{2} \\
& +\sqrt{\left(R^{2}+d^{2}+\left|\boldsymbol{a}_{0}+\boldsymbol{r}\right|^{2}\right)^{2}-4 R^{2}\left|\boldsymbol{a}_{0}+\boldsymbol{r}\right|^{2}},
\end{aligned}
$$

where $a_{0}$ is the distance between the QD and the ellipsoid centers. (See Fig. 1.) We emphasize that the induced charge depends only on the coordinate $\xi_{r}$ of the external charge, as is readily seen from Eq. (1). This is one of the crucial points for the experimental realization of the qubit-qubit coupling. Thus, positioning the QD below the gate as in previous setups [11] is not useful for the qubit-qubit coupling proposed here, since $\left(\partial q_{\text {ind }} / \partial \boldsymbol{r}\right)_{\mid r=0} \approx 0$. This fact can be exploited, however, to turn on and off the effective coupling between the qubits. Alternatively, one can use a switch that interrupts the charge-displacement current through the floating gate and thus disables the buildup of charge gradients at the far disc.

Figure 2 depicts both the induced charged $q_{\text {ind }}$, as well as the charge variation $\left(\partial q_{\text {ind }} / \partial x\right)_{\mid r=0}$ as a function of the horizontal distance $a_{0}$ between the center of the QD and the center of the gate. We see that, for very small vertical distances, $d \ll R$, the variation of the induced charge peaks at $a_{0} \approx R$, reaching values as high as unity for $d=$ $0.1 R$, and falls quickly for $a_{0}$ larger or smaller than $R$. As mentioned above, this characteristic could be used as an efficient switching mechanism. However, as $d$ increases to higher values, comparable to the disc radius $R$, the charge variation $\left(\partial q_{\text {ind }} / \partial x\right)_{\mid r=0}$ flattens out over a wide range of in-plane distances $a_{0}$. This means that, for larger depths, $d \geqslant \lambda$, of the quantum dot, the switching mechanism turns out to be rather inefficient, even though the magnitude of the coupling is only weakly reduced $\left(\left(\partial q_{\text {ind }} / \partial x\right)_{\mid r=0} \approx 0.3\right.$ for $a_{0} \approx R$ and $d=0.5 R$ ). Nevertheless, the gates confining the QDs, as well as the 2DEG itself, could lead to screening of the interaction between the QD and the floating gate, allowing for an improved switching even in this case $(d \geqslant \lambda)$.

Finally, by utilizing the expression for the electrostatic potential of a charged thin disc [20], we arrive at the expression for the electrostatic coupling,

$$
V\left(\boldsymbol{r}_{1}, \boldsymbol{r}_{2}\right)=\frac{\pi \alpha_{q}}{\kappa} \frac{e^{2} q_{\text {ind }}\left(\boldsymbol{r}_{1}\right) q_{\text {ind }}\left(\boldsymbol{r}_{2}\right)}{R},
$$

where $\kappa$ is the dielectric constant, $\alpha_{q}=\frac{C_{\mathrm{d}}}{C_{\mathrm{w}}+2 C_{\mathrm{d}}}$ is the charge-distribution factor of the gate, and $C_{\mathrm{d}}$ and $C_{\mathrm{w}}$ are the capacitances of the discs and wire, respectively. (See Appendix A.) We mention that Eq. (3) is derived in the limit when the floating gate is immersed in the dielectric. It provides a lower bound for $V\left(\boldsymbol{r}_{1}, \boldsymbol{r}_{2}\right)$ in the realistic case when the floating gate sits on top of the dielectric. 

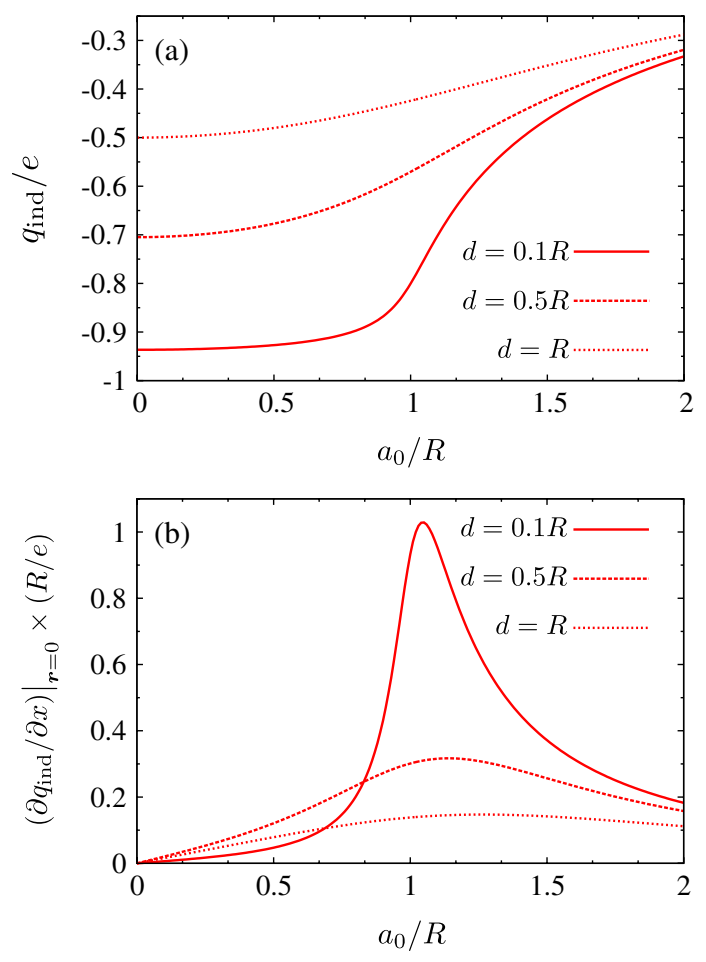

FIG. 2. The dependence (a) of the induced charge, $q_{\text {ind }}$, and (b) of the derivative of the induced charge, at $r=0$, $\left(\partial q_{\text {ind }} / \partial x\right)_{\mid r=0}$, on $a_{0}$, which is the in-plane distance from the center of the cylindrical gate to the center of the QD. We plot these two quantities for several vertical distances $d$ between the QD and the gate: $d / R=0.1,0.5,1$, corresponding to the solid, dashed, and dotted lines, respectively.

\section{QUBIT-QUBIT COUPLING}

Next, we consider the coupling between qubits. These can be for either single or double QDs. The two-qubit system with the floating gate is well described by the Hamiltonian

$$
H=V+\sum_{i=1,2} H_{\text {qubit }}^{i}
$$

where $V$ describes the electrostatic coupling between the distant charges in the qubits and is given by Eq. (3), and $H_{\text {qubit }}^{i}$ stands for either the single-QD or double-QD (DQD) Hamiltonian [17,21]:

$$
\begin{gathered}
H_{\mathrm{QD}}=H_{0}+H_{\mathrm{Z}}+H_{\mathrm{SO}}, \\
H_{\mathrm{DQD}}=J\left(\boldsymbol{S}_{1} \cdot \boldsymbol{S}_{2}\right)+H_{\mathrm{Z}}^{1}+H_{\mathrm{Z}}^{2} .
\end{gathered}
$$

Here, $H_{0}=p_{i}^{2} / 2 m^{*}+m^{*}\left(\omega_{x}^{2} x_{i}^{2}+\omega_{y}^{2} y_{i}^{2}\right) / 2$ is the energy of an electron in dot $i$ described by a harmonic confinement potential, with $m^{*}$ being the effective mass and $\hbar \omega_{x, y}$ being the corresponding single-particle-level spacings. For a single QD [Eq. (5)], $H_{\mathrm{Z}}=g \mu_{B} \boldsymbol{B} \cdot \boldsymbol{\sigma} / 2$ stands for the Zeeman coupling, with $\boldsymbol{\sigma}$ being the Pauli matrix for the spin $-\frac{1}{2}$. Both the Rashba and the Dresselhaus spin-orbit interactions are given by

$$
H_{\mathrm{SO}}=\alpha\left(p_{x} \sigma_{y}-p_{y} \sigma_{x}\right)+\beta\left(-p_{x} \sigma_{x}+p_{y} \sigma_{y}\right),
$$

where $\alpha(\beta)$ is the Rashba (Dresselhaus) spin-orbit interaction strength. The double QD is described by an effective Heisenberg model [21], Eq. (6), with $\boldsymbol{S}_{i}(i=1,2)$ being the spin in the double QD. In what follows, we assume the floating gate to be aligned along the $x$ axis; see Fig. 1.

\section{A. Singly occupied double QDs}

We start by considering two single-QD qubits. Let us first give a physical description of the qubit-qubit coupling. The purely electrostatic coupling between the QDs involves only the charge degrees of freedom of the electrons. Within each QD, the spin degree of freedom is then coupled to the charge degree of freedom via spin-orbit interaction. Hence, we expect the effective spin-spin coupling to be second order in the SOI and first order in the electrostatic interaction. In fact, one has also to assume Zeeman splitting to be present on at least one QD in order to remove the Van Vleck cancellation [22,23]. Such a cancellation occurs due to linearity in the momentum of the SOI-for the SOI cubic in the momentum (as for the self-assembled QDs, for example), one obtains a spinspin coupling even in the absence of magnetic fields [24].

Proceeding to a quantitative description, we assume the spin-orbit strength to be small compared to the QD confinement energies $\hbar \omega_{x, y}$. Following Refs. [17,23], we apply a unitary Schrieffer-Wolff (SW) transformation to remove the first-order SOI terms. The resulting Hamiltonian has decoupled spin and orbital degrees of freedom (to second order in SOI), with the effective qubit-qubit coupling (described in Appendix A), with

$$
\begin{gathered}
H_{\mathrm{S}-\mathrm{S}}=J_{12}\left(\boldsymbol{\sigma}_{1} \cdot \gamma\right)\left(\boldsymbol{\sigma}_{2} \cdot \gamma\right), \\
J_{12}=\frac{m^{*} \omega_{x, 12}^{2} E_{\mathrm{Z}}^{2}}{2\left(\omega_{x}^{2}-E_{\mathrm{Z}}^{2}\right)^{2}}
\end{gathered}
$$

where $\gamma=(\beta \cos 2 \gamma,-\alpha-\beta \sin 2 \gamma, 0)$, and $\gamma$ is the angle between the crystallographic coordinate axis along the [100] direction and the $\mathrm{x}$ axis of the coordinate system shown in Fig. 1. Here, we assume for simplicity that the magnetic field is perpendicular to the $2 \mathrm{DEG}$ substrate, with $E_{\mathrm{Z}}=g \mu_{B} B$ being the corresponding Zeeman energy. (We also assume the same perpendicular field for both dots.) However, neither the orientation nor the possible difference in the Zeeman splittings in the two dots affects the functionality of our scheme. (See Appendix A for the most general coupling case.) We note that the spin-spin interaction in Eq. (8) is of Ising type, which, together with singlequbit gates, forms a set of universal gates, as discussed in Sec. V.

All information about the floating-gate coupling is embodied in the quantity 


$$
\omega_{x, 12}^{2}=\pi \alpha_{q} \alpha_{\mathrm{C}}\left(\frac{\partial q_{\text {ind }}}{\partial \tilde{x}}\right)_{\mid r=0}^{2} \omega_{x}^{2}
$$

where $\alpha_{\mathrm{C}}=e^{2} /\left(\kappa R \hbar \omega_{x}\right)$ and $\tilde{x}=x / \lambda$, with $\lambda$ being the QD size [25]. Remarkably, the coupling has only a weak dependence on the wire length $L$ - through the capacitance ratio $\alpha_{q}$.

Next, we give estimates for the qubit-qubit coupling for GaAs and InAs QDs. Taking the spin-orbit strength for GaAs semiconductors $\lambda / \lambda_{\mathrm{SO}} \simeq 10^{-1}\left(\lambda_{\mathrm{SO}}=\hbar /\left(m^{*} \alpha\right)=\right.$ $2 \mu \mathrm{m})$, and assuming that $E_{\mathrm{Z} 1} \simeq E_{\mathrm{Z} 2} \equiv E_{\mathrm{Z}} \simeq 0.5 \hbar \omega_{x}$ (with $B=2 T$ and $\hbar \omega_{x} \simeq 1 \mathrm{meV}$ ), we obtain $H_{\mathrm{S}-\mathrm{S}} \simeq$ $\alpha_{q} \alpha_{\mathrm{C}}\left(\partial q_{\text {ind }} / \partial \tilde{x}\right)_{\mid r=0}^{2} \times 10^{-7} \mathrm{eV}$. The electrostatic coupling strongly depends (as $d^{-2}$ does) on the vertical distance between the gate and the QDs. Typically, $d \simeq \lambda$, and one obtains, using Eq. (1), maximal coupling $H_{\mathrm{S}-\mathrm{S}} \simeq$ $10^{-11}-10^{-10} \mathrm{eV}$ (for $R=1.6 \lambda, L=10 \mu \mathrm{m}$, and $R_{\mathrm{w}}=$ $30 \mathrm{~nm}$, leading to $\alpha_{q}=0.02 ; a_{0}=1.9 \lambda$ ). Although it is experimentally challenging to decrease $d$ to a value of about $10 \mathrm{~nm}$, the gain would be a significantly stronger coupling of $10^{-9}-10^{-8} \mathrm{eV}$ (for $R=0.17 \lambda$ and $a_{0}=0.2 \lambda$ ). Moreover, if a semiconductor with larger spin-orbit coupling is used-such as InAs $\left(\lambda / \lambda_{\text {SO }} \simeq 1\right)$-the coupling is increased by 2 orders of magnitude compared to that of $\mathrm{GaAs}$, reaching the $\mu \mathrm{eV}$ regime. Quite remarkably, these values almost reach within the exchange-strengths range, $J_{\text {exc }} \sim 10-100 \mu \mathrm{eV}$ (10-100 ps), that occurs in typical GaAs double quantum dots [1,4]. Actually, for realistic devices as presented in the Sec. VI, the coupling is almost 2 orders of magnitude larger than the estimates presented here, and thus operation times are well below the decoherence times for QD. This discrepancy is not very surprising, and it is mainly due to our conservative treatment of the dielectric and the sensitivity of the electric-field gradient to the geometry of the surrounding gates.

\section{B. Hybrid spin qubits}

A number of different spin-based qubits in quantum dots have been investigated over the years [26], each with its own advantages and challenges. The most prominent ones are spin $-\frac{1}{2}$ and singlet-triplet spin qubits. Here, we show that these qubits can be crosscoupled to each other, thus forming hybrid spin qubits which open up the possibility to take advantage of the best of both worlds.

We model the hybrid system by a single- and a doubleQD qubit, described by Eqs. (5) and (6), respectively. The single QD and the floating gate act as an electric field, leading to the change in the splitting between the logical states of the double-QD spin qubit, $J \rightarrow J+\tilde{x}_{\mathrm{e}} \delta \tilde{J}$ [21], with $x_{\mathrm{e}}=\tilde{x}_{\mathrm{e}} \lambda$ being the $x$ coordinate of the electron in the single QD and

$$
\delta \tilde{J}=\frac{3}{\sinh (2 \tilde{l})} \frac{\omega_{x, 12}^{2}}{\tilde{l} \omega_{\mathrm{D}}^{2}} \epsilon .
$$

Here, $\omega_{\mathrm{D}}$ is the confinement energy in the $\mathrm{DQD}$, and $\tilde{l}$ is the distance between the double-QD minima measured in units of a QD size $\lambda$. The previous formula is valid for the regime $\epsilon \gtrsim \omega_{\mathrm{D}}$.

In order to decouple spin and orbital degrees of freedom, we again employ a Schrieffer-Wolff transformation and obtain the hybrid coupling in the lowest order in SOI (see Appendix B):

$$
H_{\text {hybrid }}=\frac{3 \mu \mathrm{g} \delta \tilde{J}(\boldsymbol{\gamma} \times \boldsymbol{B}) \cdot \boldsymbol{\sigma}}{4\left(\omega_{x}^{2}-E_{1 Z}^{2}\right) \lambda} \tau_{z} .
$$

Here, $\tau_{z}$ is a Pauli matrix acting in the pseudospin space spanned by the logical states of the singlet-triplet qubit. It should be noted that the sign of this coupling can be manipulated by changing the sign of the detuning voltage $\epsilon$. As an estimate, we can write

$$
H_{\text {hybrid }} \simeq\left(\frac{\omega_{x, 12}}{\omega_{x}}\right)^{2} \frac{E_{\mathrm{Z}}}{\omega_{\mathrm{D}}} \frac{a_{B}}{\lambda_{\mathrm{SO}}} \epsilon .
$$

Assuming the parameters given in the previous section for the GaAs QDs, we obtain the estimate $H_{\text {hybrid }} \simeq$ $10^{-10}-10^{-9} \mathrm{eV}$. By reducing the distance $d$ or using InAs QDs, we can gain 1 order of magnitude more in the coupling.

\section{Doubly occupied double QDs}

To complete our discussion about the qubit-qubit couplings, we now consider two double QDs coupled via the floating gate. As already noted, owing to the different charge distributions of the logical states in the double QD, the SOI term is not needed for the qubit-qubit coupling [18]. Certainly, the SOI exists in double QDs, but its effect on the singlet-triplet (ST) splitting can be neglected [27]. Here, only a rough estimate of the coupling is provided, while the detailed analysis can be found in Ref. [18].

We assume both double QDs to be strongly detuned, so the singlet logic state is almost entirely localized on the lower potential well of the double QD. The electrostaticenergy difference between the singlet-singlet and triplettriplet system configurations gives the rough estimate of the qubit-qubit coupling, $H_{\mathrm{S}-\mathrm{S}} \simeq V(R, R)-V(R+l$, $R+l)$. Taking the distance between the double-QD minima $l \simeq R$ and the same GaAs parameters as before, we finally obtain the estimate $H_{\mathrm{S}-\mathrm{S}} \simeq 10^{-5}-10^{-6} \mathrm{eV}$. As can be seen from Fig. 2, reducing $d$ to $10 \mathrm{~nm}$ increases the coupling by a factor of 5 .

\section{SCALABLE ARCHITECTURE}

One central issue in quantum computing is scalability, meaning that the basic operations such as initialization, readout, and single-qubit and two-qubit gates should not depend on the total number of qubits. In particular, scalability enables the implementation of fault-tolerant 
quantum-error-correction [3], such as surface codes where error thresholds are as large as $1.1 \%[5,6]$.

For such implementations, the architecture of the qubit system becomes of central importance [28]. Making use of the electrostatic long-distance gates presented above, we now discuss two illustrative examples for such scalable architectures.

\section{A. Design with floating metal gates}

In the first design we propose here, the metallic gates above the 2DEG are utilized for qubit-qubit coupling, while the switching of the coupling is achieved by moving the QDs (see Fig. 3). Only the coupling between adjacent QDs is possible in this design. Without this constraint, the induced charge due to nearby QDs would be spread over the whole system, resulting in an insufficient qubit-qubit coupling.

The actual virtue of the setup is its experimental feasibility, as suggested by recent experiments [11,12]. However, as explained in Sec. II, a minor but crucial difference here is that the qubit-qubit coupling depends not on the charge itself but rather on its gradient, in contrast to earlier designs $[11,12]$. This dependence requires the dots to be positioned off the disc-center.

In order to complete our quantum-computer design, we have to equip our system with a fast switch. The discussion in Sec. II becomes relevant therefore, because the coupling can be turned off (on) by moving a QD away (toward) the corresponding floating gate. (See Fig. 2.) The spatial change of the quantum dot induces an electric response

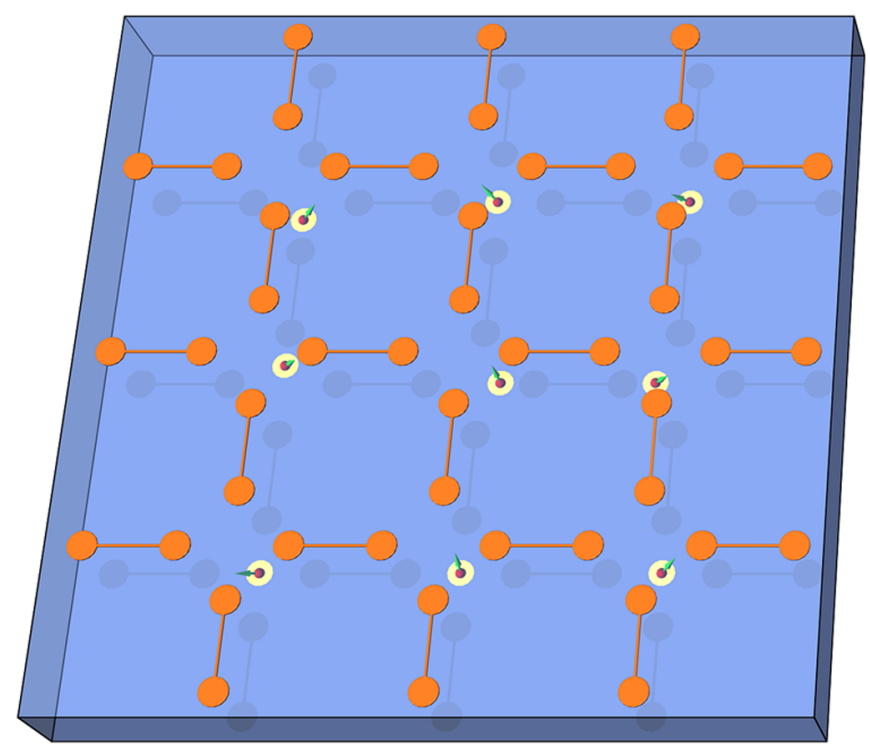

FIG. 3. Quantum-computer architecture using metallic floating gates on top of a 2DEG. The electrostatic long-range coupling is confined to adjacent qubits. Turning on (off) the qubit-qubit interaction is achieved by moving a qubit close to (away from) the corresponding metal disc. This architecture allows for parallel switching. in the metallic floating gate on a time scale roughly given by the mean free time of the elastic scattering (at low temperatures). This is the time it takes to reach the new electronic-equilibrium configuration that minimizes the electrostatic energy. Since, for a typical metal, this response time is on the order of tens of femtoseconds, it poses no limitations, being much faster than the effective switching times obtained in the previous sections.

\section{B. All-in-2DEG design}

We now consider a setup in which all elements of the qubit network, including the floating connector gates, are implemented in the 2DEG itself. This arrangement allows us to extend the above design in an essential way, namely, to implement a switching mechanism inside the connectors themselves which is potentially fast and efficient (with a large on/off ratio). Two attractive features come with such a design: First, the qubit-qubit coupling is now controlled by the connector switch only, while the quantum dots with

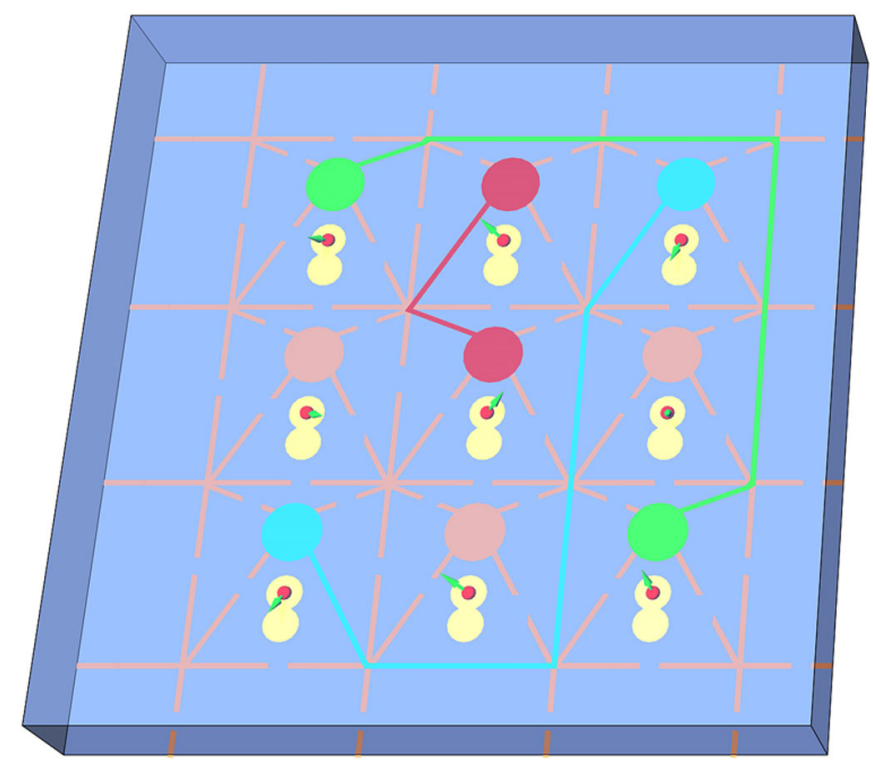

FIG. 4. All-in-2DEG design: The qubits and the floating connector gates are all implemented within the same 2DEG. The spin qubits (green arrows) are confined to double quantum dots (small yellow double circles) and are each at a fixed position with maximum coupling strength to the floating gate (large disc). (See Fig. 2.) The network consists of quantum channels (the colored lines) that enable the electrostatic coupling between discs (large circles) so that two individual qubits at or beyond nearest-neighbor sites can be selectively coupled to each other. In the figure, three pairs of particular discs are shown, connected by quantum channels (solid lines), while the remaining discs (beige) are disconnected from the network (interrupted beige lines). The large discs can be considered as large quantum dots that contain many electrons. The quantum wires can be efficiently disconnected (as in the interrupted lines) by depleting the single channel with a metallic top gate (not shown). This architecture allows for parallel switching. 
the spin qubits can be left fixed, thereby reducing the source of gate errors. Second, this design allows for coupling beyond nearest-neighbor qubits, which is beneficial for the error threshold in fault-tolerant quantum error correction schemes [28].

The proposed network is shown in Fig. 4, where the floating gates are formed within the 2DEG in the form of discs connected by quantum wires. The discs themselves can be considered as large quantum dots that contain many electrons (approximately 50-100) so that (quantum) fluctuations are negligibly small. Parts of the network are then connected or disconnected by locally depleting these wires with the help of a standard quantum point contact [1]. This suppresses the displacement of charges very quickly and efficiently. The electrostatics of such semiconductor gates is essentially the same as the previously discussed metallic gate. Indeed, the number of electrons in the 2DEG-defined network can be fixed, thus the gate behaves as floating. Again, the minimal switching time is limited roughly by the mean free time of the elastic scattering (at low temperatures), which for a typical GaAs 2DEG is on the order of tens of picoseconds.

The single-spin control required for completing the universal set of gates in our proposal can be implemented in both setups through ESR [29], or purely electrically via electric-dipole-induced spin resonance [30-32], which is more convenient for our electrostatic scheme. The time scales achieved are on the order of $50 \mathrm{~ns}$, much shorter than the spin-relaxation and decoherence times [30-32].

\section{Design based on 1D-nanowire quantum dots}

The efficiency of the floating-gate architecture is strongly dependent on the strength of the SOI experienced by the electrons in the QDs, which has to be large enough to overcome the spin-decoherence rates. InAs nanowires are such strong SOI materials, with strengths larger by an order of magnitude than in GaAs 2DEG [33]. Moreover, the electron spins in QDs created in these nanowires show long coherence times [32] and can be controlled (electrically) on times scales comparable to those found for the electron-spin manipulation in GaAs gate-defined QDs [32].

In Fig. 5, we show a sketch of an architecture based on nanowires that contain single or double QDs. Typical examples for such wires are InAs [32,33] and $\mathrm{Ge} / \mathrm{Si}$ $[13,34]$ nanowires, carbon nanotubes [12,35-37], etc. The default position of a QD is chosen so that the coupling to any of the surrounding gates is minimal. Neighboring QDs in the same nanowire are coupled by a vertical metal gate, while QDs in adjacent nanowires are coupled by a horizontal metal gate. The electron in a given QD can be selectively coupled to only two of the surrounding gates by moving it (via the gates that confine the electrons) in regions where the electric-field gradient for the induced charge is maximum on these two "active" gates, while negligible for the other two "passive" gates. The other QD

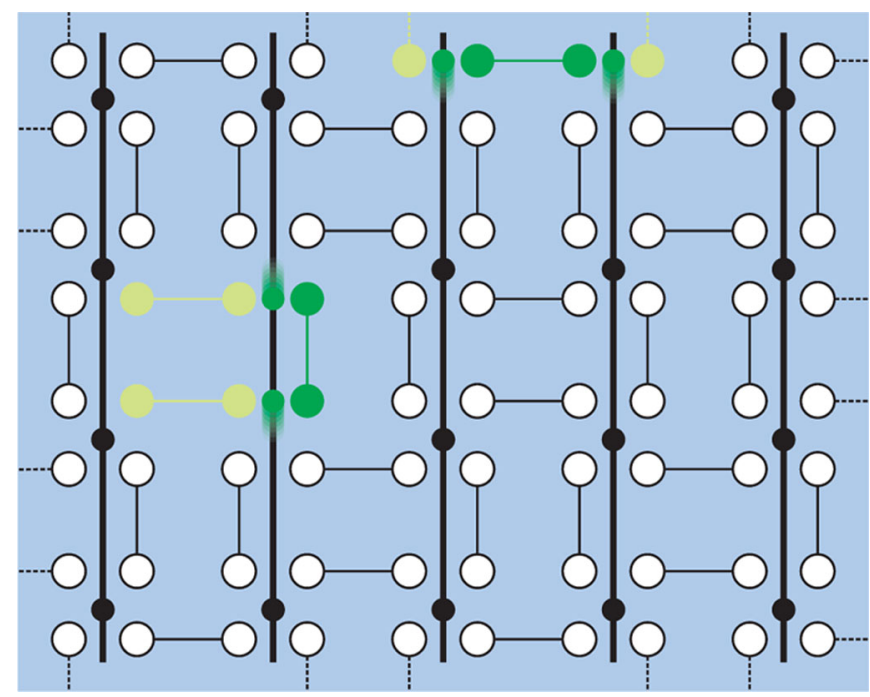

FIG. 5. Architecture based on nanowire QDs coupled by metallic gates. The spin qubits are confined to QDs (black dots) on nanowires. The nanowires form a parallel array (vertical black lines). The coupling between neighboring spin qubits is enabled by floating metal gates (white circles) positioned either parallel to the wires (and thus coupling QDs created in the same wire), or perpendicular to the wires (and thus coupling QDs created in adjacent nanowires). By using external gates (not shown) to move the dots along the nanowires (shaded green colors on the wires), it is then possible to selectively couple one particular QD to only two surrounding gates (green and yellow "active" gates). The other QD partner also couples to one of these active gates (green), resulting in a selective coupling of the desired nearestneighbor pair.

partner in the coupling is moved toward one of the active gates, resulting in a qubit-qubit coupling. Note that there are three active gates in total, but only one of them is shared by both QDs. This configuration allows selective coupling of any nearest-neighbor pair in the network.

The spin-coupling mechanism as well as the 2D geometry are similar to the previous 2DEG-GaAs-QD designs, showing the great flexibility of the floating-gate architecture. As before, the spin qubits can be manipulated purely electrically, via the same gates that confine the QDs [32]. We mention also that the (dog-bone-like) gate geometry shown in Fig. 5 is not optimized to achieve the best switching ratio; more asymmetric gate geometries may possibly lead to better results.

\section{Spin-qubit decoherence and relaxation}

Decoherence and relaxation are two of the main obstacles to overcome in building a quantum computer. The main source of qubit decay in typical GaAs quantum dots comes from nuclear spins and phonons (via spin-orbit interaction), and it has been studied in great detail both theoretically and experimentally. (See, e.g., Ref. [38].) The longest relaxation and decoherence times measured are about $T_{1} \sim 1 s$ [39] and $T_{2} \sim 270 \mu \mathrm{s}$ [2], respectively. 
Exactly the same qubit-decay mechanisms as described also apply here except for one new source that comes from the Nyquist noise of the floating metallic gates. However, this problem has been studied in great detail in Ref. [40], and no major impact on the decoherence time has been found. Even if Nyquist noise were a problem, it could be further reduced by using superconducting gates in lieu of normal metal ones.

\section{IMPLEMENTATION OF TWO-QUBIT GATES}

Since the Hamiltonian of Eq. (8) can entangle, it can be used to implement two-qubit gates. Here, we consider the controlled-NOT (CNOT) gate, widely used in schemes for quantum computation [5,6]. The Hamiltonian for two single-QD qubits interacting via the floating gate is the sum of $H_{\mathrm{S}-\mathrm{S}}$ and the Zeeman terms. The strength of the latter in comparison to the former allows us to approximate the Hamiltonian by $H^{\prime}=J_{12}|\boldsymbol{\gamma}|^{2}\left(\sigma_{x}^{1} \sigma_{x}^{2}+\sigma_{y}^{1} \sigma_{y}^{2}\right) / 2+$ $E_{z}\left(\sigma_{z}^{1}+\sigma_{z}^{2}\right) / 2$, for which the qubit-qubit interaction and Zeeman terms commute. The CNOT gate, $C$, may then be realized with the following sequences,

$$
\begin{aligned}
C= & \sqrt{\sigma_{z}^{1}} \sqrt{\sigma_{x}^{2}} \mathcal{H}^{1} e^{i\left(\sigma_{z}^{1}+\sigma_{z}^{2}\right) E_{z} t} e^{-i H^{\prime} t} \\
& \times \sigma_{x}^{1} e^{i\left(\sigma_{z}^{1}+\sigma_{z}^{2}\right) E_{z} t} e^{-i H^{\prime} t} \sigma_{x}^{1} \mathcal{H}^{1}, \\
C= & \sqrt{\sigma_{z}^{1}} \sqrt{\sigma_{x}^{2}} \mathcal{H}^{1} \sigma_{x}^{2} e^{-i H^{\prime} t / 2} \sigma_{x}^{1} \sigma_{x}^{2} e^{-i H^{\prime} t / 2} \\
& \times \sigma_{x}^{2} e^{-i H^{\prime} t / 2} \sigma_{x}^{1} \sigma_{x}^{2} e^{-i H^{\prime} t / 2} \mathcal{H}^{1},
\end{aligned}
$$

where $t=\pi /\left(4 J_{12}\left|\gamma_{x}\right|^{2}\right)$ and $\mathcal{H}$ denotes the single-qubit Hadamard rotation. These sequences require two and four applications of the floating gate, respectively. More details on their construction can be found in Appendix C. The time $t$ is the bottleneck process in the sequence, and so the time needed to implement the gates is on the order of this value. For a realistic value of $J_{12}|\gamma|^{2}=10 \mu \mathrm{eV}$, this gives a time of around a nanosecond.

Since $H^{\prime}$ is only an approximation of the total Hamiltonian, these sequences yield approximate CNOTs. Their success can be characterized by the fidelity which depends only on the relative strengths of the parameters. For a realistic device, we can expect the Zeeman terms to be an order of magnitude stronger than the qubit-qubit coupling. The above sequences then yield fidelities of $99.33 \%$ and $99.91 \%$, respectively. For 2 orders of magnitude between the Zeeman terms and qubit-qubit coupling, the approximation improves, giving fidelities of $99.993 \%$ and $99.998 \%$, respectively. These are all well above the fidelity of $99.17 \%$ that corresponds to the threshold for noisy CNOTs in the surface code [6]. Hence, despite the difference in error models, we can be confident that the gates of our scheme are equally useful for quantum computation.

\section{NUMERIC MODELING OF REALISTIC DEVICES}

In the previous sections, we neglected a number of practical concerns related to the construction of working devices, most notably, the existence of the metallic gates used to define the quantum dots themselves and the presence of undepleted 2DEG outside of the quantum dots. The gates and 2DEB have finite capacitances to the coupler, shunting away some of the charge that would otherwise contribute to the interqubit interaction. To confirm that substantial couplings can still be attained at large distances with these limitations, we have performed numeric simulations of devices with realistic geometries similar to ST
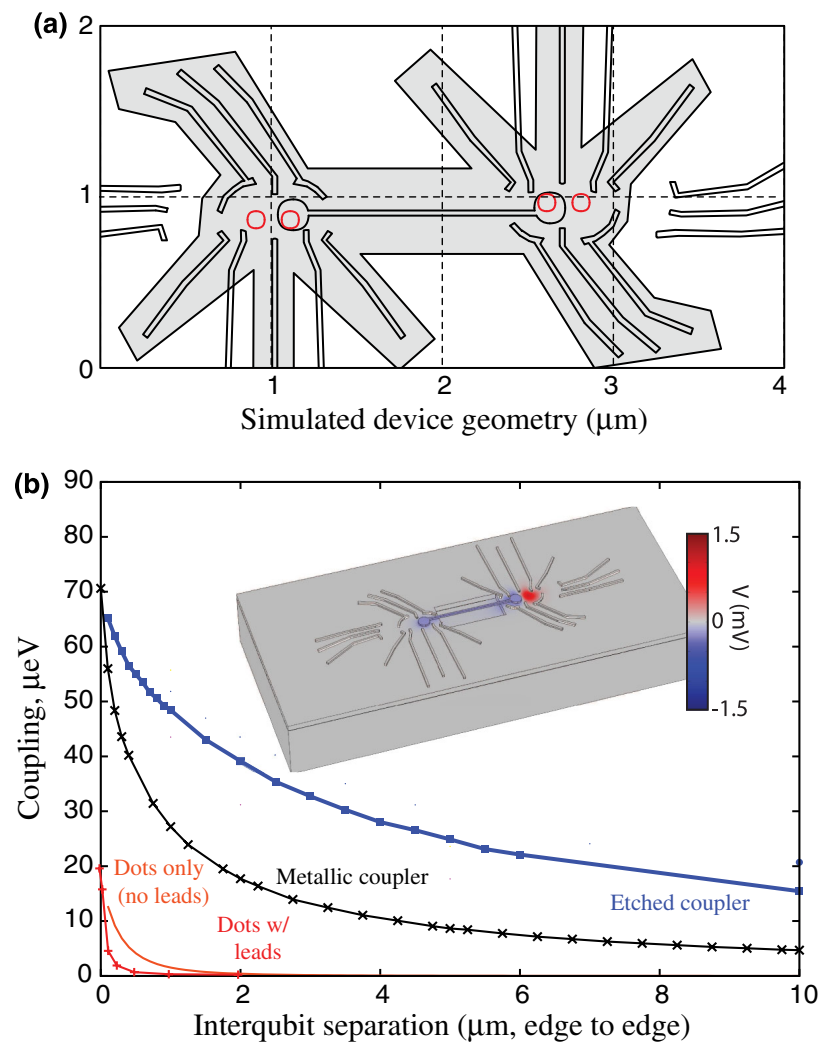

FIG. 6. Numeric simulation confirms the efficacy of the design for ST qubits; addition of a metallic coupler (crosses) increases coupling more than threefold for closely spaced dots, and greatly extends the range of the coupling. (a) The simulated device with a separation of $1 \mu \mathrm{m}$ and an etched coupler. 2DEG underneath the shaded region is treated as depleted, while red circles show the locations of the individual quantum dots within the simulation. (b) Coupling strength as a function of separation for the ST qubits in free space (smooth red curve, second from lower left), qubits including leads and 2DEG but without a coupler (red curve with + signs in lower left corner), including a metallic coupler (curve with black crosses), and additionally etching a trench around the coupler to deplete the 2DEG underneath (curve with blue squares). Inset: Electrostatic potential (color scale) at the sample surface shows the impact of the coupler on a device with a $1-\mu \mathrm{m}$ separation. 
spin qubits that are currently in use. A typical simulated geometry is included in Fig. 6. The gate and heterostructure design is identical to a functional device currently being characterized [41], and the boundaries of the 2DEG and placement of the electrons within the dot are estimates guided by experimentally measured parameters. Each quantum dot is modeled as a fixed-charge metallic disc $50 \mathrm{~nm}$ in diameter within the 2DEG. While unsophisticated, the simulation suffices to estimate the practicality of this scheme.

We define the coupling between two ST qubits as the change in detuning in one ST qubit induced by the transfer of a full electron from one dot to the other dot in a second ST qubit. For our reference ST-qubit design with the two qubits physically adjacent to each other and no coupler (680 $\mathrm{nm}$ center to center), we calculate a coupling of
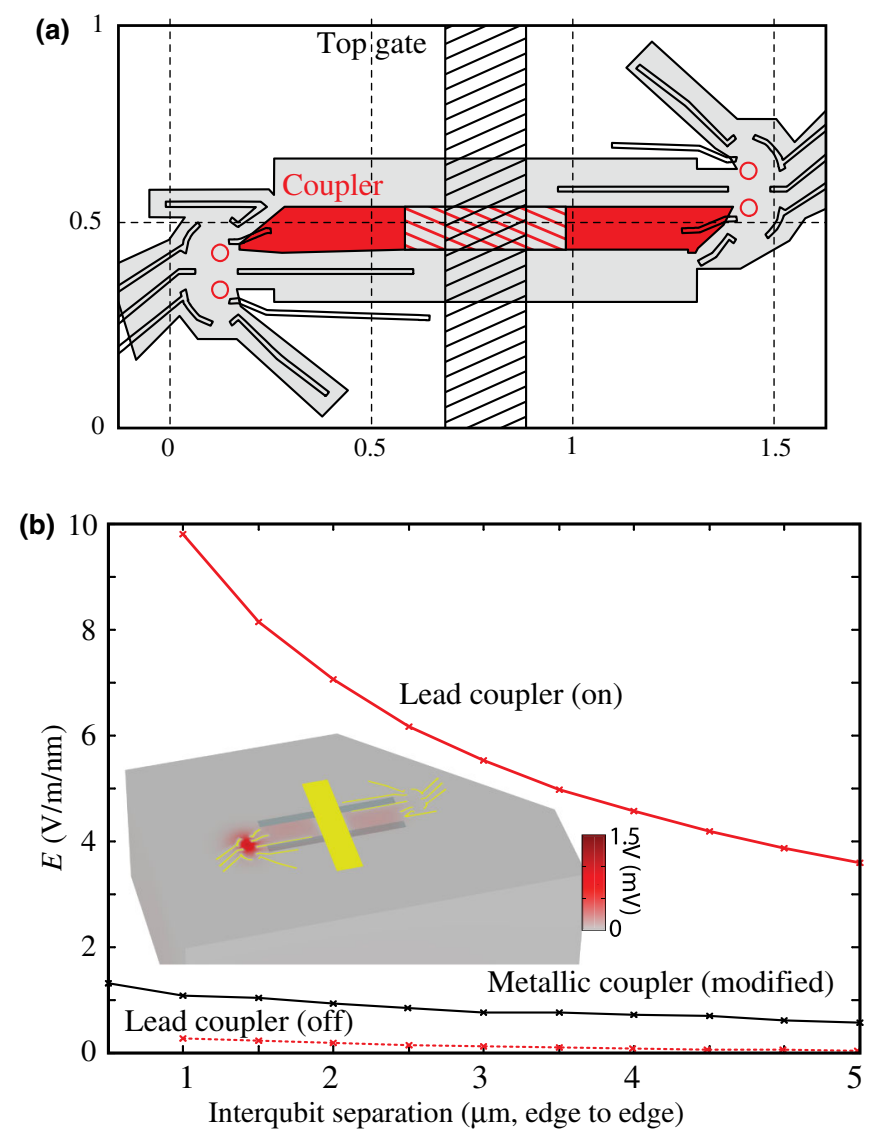

FIG. 7. Simulations of single-spin qubits show appreciable coupling strengths, even over distances of several microns. While the metallic coupler design of Fig. 6 modified to place the quantum dots at the edges of the couplers is effective [black crosses in (b)], an all-in-2DEG design where one of the leads of the qubit acts as a coupler [red region in (a)] provides dramatically enhanced coupling [solid red lines in (b)]. The coupler is deactivated by a metallic top gate [black hatched region in (a)], modeled by removing the hatched section of the coupler. The deactivation reduces interqubit coupling by over an order of magnitude [dashed red curve in (b)].
$20 \mu \mathrm{eV}$. As the qubits are separated, the coupling vanishes rapidly as the 2DEG between the qubits screens the electric field; the coupling is reduced by an order of magnitude if the dots are separated by an additional $250 \mathrm{~nm}$. This rapid falloff makes problematic the gate density needed for large-scale integration of these qubits.

The addition of a floating metallic coupler of the type described here increases the coupling at zero separation to $70 \mu \mathrm{eV}$ and allows the qubits to be separated by more than $6 \mu \mathrm{m}$ before the coupling drops to the level seen for two directly adjacent qubits. We can further improve upon this coupling by etching the device in the vicinity of the coupler, which reduces the shunt capacitance of the coupler to the grounded 2DEG between the devices.

For the case of single spins, this metallic coupler is modified to place the quantum dots at the edges of the coupler rather than under the discs. We define the coupling in this case as the electric field in $\mathrm{V} / \mathrm{m}$ induced on one qubit in response to $1 \mathrm{~nm}$ of motion of the electron on the other qubit. We continue to find substantial couplings even at large separations (Fig. 7). However, in this case we find we can further improve couplings by moving to the all-in2DEG design, where one of the leads of the quantum dot is used as the coupler [Fig. 7(a)]. Using the lead in this fashion should be harmless; no current is driven into the lead during qubit manipulations. The lead [colored region in Fig. 7(a)] is modeled as a metallic strip at the level of the 2DEG. Because of the close proximity of the lead to the qubit as well as the sharp electric-field gradients near the point of the lead, we find strongly enhanced coupling for this lead coupler over the floating metallic coupler for single-spin qubits. By depleting part of the lead coupler using a metallic top gate [yellow region in Fig. 7(b)], it is possible to selectively turn this coupling on and off. The reduction in coupling in the off state is more than an order of magnitude, and that can be further improved by increasing the size of the depleted region.

\section{CONCLUSIONS}

We have proposed and analyzed an experimentally feasible setup for implementing quantum gates in an array of spin qubits localized in gate-defined quantum dots based on the interplay of the Coulomb repulsion between the electrons, SOI, and externally applied magnetic fields. As opposed to current schemes based on direct exchange [4], our scheme does not require electron tunneling between the quantum dots and thus is within experimental reach based only on current spin-qubit technology.

We have shown, both analytically and numerically, that by use of either metallic floating gates in the shape of a dog bone, or the 2DEG itself acting as a metallic gate, longrange spin-spin coupling is achieved, with coupling strengths exceeding the spin-decay rates. Moreover, the coupling can be selectively switched on and off between any pairs of qubits by only local qubit manipulation, 
allowing entangling quantum gates such as the CNOT to be performed accurately and efficiently. The two-dimensional architecture based on this design provides a platform for implementing the powerful surface code.

The electrostatic scheme proposed here is also a step forward toward an efficient implementation of gates between hybrid qubits, such as the ST qubit, hole-spin qubits, or even superconducting qubits. This work opens up new avenues for a future working hybrid quantum computer, based on not one but several types of qubits.

\section{ACKNOWLEDGMENTS}

We thank C. Marcus, S. Gangadharaiah, and D. Stepanenko for helpful discussions. We acknowledge support from the Swiss NSF, the NCCR's Nanoscience and QSIT, and DARPA. This research was partially supported by the IARPA/MQCO program and the U. S. Army Research Office under Contract No. W911NF-11-1-0068. M. T. acknowledges financial support from the NSF under Grant No. DMR-0840965.

\section{APPENDIX A: SPIN-SPIN COUPLING-SINGLY OCCUPIED DOUBLE QUANTUM DOTS}

In this section, we derive explicitly the effective spin-spin coupling. The spin-orbit interaction (SOI) Hamiltonian $H_{\mathrm{SO}}$ is assumed to be small compared to both the orbital Hamiltonian $H_{0}+V$ and the Zeeman coupling $H_{\mathrm{Z}}$, so that we can treat it using perturbation theory. The method of choice for perturbation theory is based on the SW transformation, following Refs. [17,23]. This method is quite suitable for deriving effective Hamiltonians, which is our goal. We first perform a unitary transformation on the full Hamiltonian, $H \rightarrow e^{S} H e^{-S} \equiv$ $H_{\mathrm{SW}}$, with $S$ an antiunitary operator, so that we obtain

$H_{\mathrm{SW}}=H_{\mathrm{d}}+H_{\mathrm{SO}}+\left[S, H_{\mathrm{d}}+H_{\mathrm{SO}}\right]+\frac{1}{2}\left[S,\left[S, H_{\mathrm{d}}+H_{\mathrm{SO}}\right]\right]+\ldots$,

where $H_{\mathrm{d}}=H_{0}+V+H_{\mathrm{Z}}$. We look for the transformation $S$ so that this diagonalizes the full Hamiltonian $H$ in the basis of $H_{\mathrm{d}}$. In leading order in $H_{\mathrm{SO}}$, we choose $S$ so that $\left[S, H_{0}+V+H_{\mathrm{Z}}\right]=-(1-\mathcal{P}) H_{\mathrm{SO}}$, with the projector operator $\mathcal{P}$ satisfying $\mathcal{P} A=\sum_{E_{n}=E_{m}} A_{n m}|m\rangle\langle n|, \forall A$. In other words, it projects onto the diagonal part of the Hamiltonian, $H_{\mathrm{d}}$. Keeping the lowest-order terms in $\alpha$ and $\beta$ in the SW transformation, we are left with the effectiveinteraction Hamiltonian $H_{\mathrm{SW}}$ that contains the desired spin-spin coupling in the basis of $H_{\mathrm{d}}$ :

$$
H_{\mathrm{SW}}=H_{\mathrm{d}}-\frac{1}{2} \mathcal{P}\left[S, H_{\mathrm{SO}}\right]
$$

where $S=(1-\mathcal{P}) L_{\mathrm{d}}^{-1} H_{\mathrm{SO}}$, with $L_{\mathrm{d}}$ being the Liouvillian superoperator $\left(L_{\mathrm{d}} A=\left[H_{\mathrm{d}}, A\right], \forall A\right)$.
Next, we find the explicit expression for the spin-spin coupling due to the second-order term in SOI in Eq. (A2), i.e., $U \equiv \frac{1}{2}\left[S, H_{\text {So }}\right]$. We make use of the explicit timedependent (integral) representation of the Liouvillian $L_{\mathrm{d}}^{-1}=-i \int_{0}^{\infty} d t e^{i\left(L_{\mathrm{d}}+i \eta\right) t}$ and arrive at

$$
U=-\frac{i}{2} \int_{0}^{\infty} d t e^{-\eta t}\left[H_{\mathrm{SO}}(t), H_{\mathrm{SO}}\right],
$$

where $H_{\mathrm{SO}}(t)=e^{i L_{\mathrm{d}} t} H_{n}=e^{i H_{\mathrm{d}} t} H_{n} e^{-i H_{\mathrm{d}} t}$, and $\eta \rightarrow 0^{+}$ensures the convergence of the time integration. Heisenberg operators, $\boldsymbol{\sigma}_{i}(t)$ and $\boldsymbol{p}_{i}(t)$, are needed in order to calculate $U$. The former is easy to obtain: $\boldsymbol{\sigma}_{i}(t)=\hat{\Sigma}_{i}(t) \boldsymbol{\sigma}_{i}$, with $\hat{\Sigma}_{i}(t)$ given by

$$
\begin{aligned}
\left(\hat{\Sigma}_{i}\right)_{m n}(t)= & \delta_{m n} l_{i}^{2} \cos \frac{E_{\mathrm{Z} i} t}{2 \hbar}+2\left(\boldsymbol{l}_{i}\right)_{m}\left(\boldsymbol{l}_{i}\right)_{n} \sin ^{2} \frac{E_{\mathrm{Z} i} t}{4 \hbar} \\
& -\varepsilon_{n m k}\left(\boldsymbol{l}_{i}\right)_{k} \sin \frac{E_{\mathrm{Zn} n} t}{2 \hbar},
\end{aligned}
$$

with $\boldsymbol{l}_{i}=\boldsymbol{B}_{i} / B$. The calculation of $\boldsymbol{p}_{i}(t)$ consists of solving the system of ordinary differential equations (ODEs):

$$
\begin{gathered}
\frac{d}{d t} \boldsymbol{p}_{i}(t)=-m^{*} \omega_{0}^{2} \boldsymbol{r}_{i}(t)-\frac{\partial}{\partial \boldsymbol{r}_{i}} V\left(\boldsymbol{r}_{1}(t), \boldsymbol{r}_{2}(t)\right), \\
\frac{d}{d t} \boldsymbol{r}_{i}(t)=\boldsymbol{p}_{i}(t) / m^{*} .
\end{gathered}
$$

In order to solve this system, we expand the electrostatic potential, given in Eq. (3), around the minima to second order in $\boldsymbol{r}_{i}(t)$. The system of ODEs now reads

$$
\begin{aligned}
& \frac{d^{2}}{d t^{2}}\left(\begin{array}{l}
\boldsymbol{p}_{1}(t) \\
\boldsymbol{p}_{2}(t)
\end{array}\right)=-\hat{\Omega}\left(\begin{array}{l}
\boldsymbol{p}_{1}(t) \\
\boldsymbol{p}_{2}(t)
\end{array}\right), \\
& \hat{\Omega}=\left(\begin{array}{cccc}
\omega_{x}^{2} & 0 & \hat{\Omega}_{12} \\
0 & \omega_{y}^{2} & & \\
\hat{\Omega}_{12}^{\dagger} & \omega_{x}^{2} & 0 \\
0 & \omega_{y}^{2}
\end{array}\right) .
\end{aligned}
$$

In this approximation, only terms $O\left(\boldsymbol{r}_{i}^{2}\right)$ are retained-This is a valid choice for low-lying levels. We ignore the renormalization of the frequencies $\left(\omega_{x}\right.$ and $\left.\omega_{y}\right)$ because it gives higher-order (in the Coulomb energy) contribution to the effective spin-spin coupling. The coupling between the QDs $\left(\hat{\Omega}_{12}\right)$ is given by

$$
\begin{gathered}
\left(\hat{\Omega}_{12}\right)_{i j}=\pi \alpha_{q} \alpha_{C}\left(\frac{\partial q_{\text {ind }}}{\partial \tilde{\boldsymbol{r}}_{i}}\right)_{\mid r=0}\left(\frac{\partial q_{\text {ind }}}{\partial \tilde{\boldsymbol{r}}_{j}}\right)_{\mid r=0} \omega_{i} \omega_{j}, \\
\left(\partial q_{\text {ind }} / \partial \boldsymbol{r}_{i}\right)_{\mid r=0}=\frac{2 R \sqrt{\xi_{0}^{2}-R^{2}} \boldsymbol{a}_{i}}{\pi \xi_{0}^{2}\left(2 \xi_{0}^{2}-a_{0}^{2}-R^{2}-d^{2}\right)},
\end{gathered}
$$

where $\alpha_{q}=C_{\mathrm{d}} /\left(C_{\mathrm{d}}+C_{\mathrm{w}}\right), \alpha_{\mathrm{C}}=e^{2} /\left(\kappa R \hbar \omega_{x}\right)$, and $\tilde{\boldsymbol{r}}_{i}=$ $\boldsymbol{r}_{i} / \lambda_{i}$. ( $\lambda_{i}$ is the QD size along the $i$ th direction). $\boldsymbol{a}_{i}$ are the vectors that define the position of the QDs with respect to 


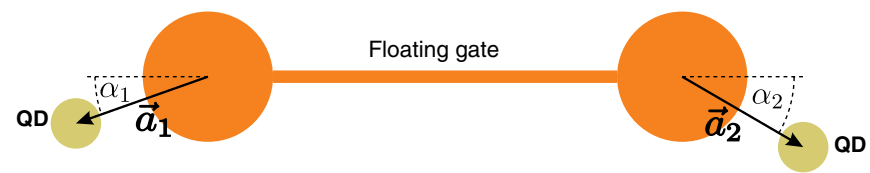

FIG. 8. The misalignment angle $\alpha$ of the two QDs (yellow discs), defined with respect to the metallic floating gate (orange).

the nearby disc center (see Fig. 8). Note that the expressions for the disc $\left(C_{\mathrm{d}}\right)$ and wire $\left(C_{\mathrm{w}}\right)$ capacitances, respectively, are given by

$$
\begin{gathered}
C_{\mathrm{d}}=2 R / \pi, \\
C_{\mathrm{w}}=\frac{L}{2 \ln \left(L / R_{\mathrm{w}}\right)},
\end{gathered}
$$

where $R$ is the radius of the disk, $R_{\mathrm{w}}$ is the radius of the wire, and $L$ is the length of the wire.

In order to obtain the solution of Eq. (A8), we note that even a slight ellipticity $\left(\left|\omega_{x}^{2}-\omega_{y}^{2}\right| \gg \max \left[\left(\hat{\Omega}_{12}\right)_{x y}^{2}\right.\right.$, $\left.\left.\left(\hat{\Omega}_{12}\right)_{y x}^{2}\right]\right)$ of the QDs causes the motion in the $x$ and $y$ direction to be decoupled. Having in mind that $\left(\hat{\Omega}_{12}\right)_{y x, x y}^{2} / \omega_{x, y}^{2} \sim 10^{-3}-10^{-4}$, we conclude that such an ellipticity is unavoidable in realistic experimental devices. Thus, we put off-diagonal elements of the $\hat{\Omega}_{12}$ matrix to zero and obtain these solutions:

$$
\begin{aligned}
\boldsymbol{p}_{1,2}^{i}(t)= & \pm \boldsymbol{p}_{a}^{i} \cos \left(\boldsymbol{\omega}_{+}^{i} t\right)+\boldsymbol{p}_{s}^{i} \cos \left(\boldsymbol{\omega}_{-}^{i} t\right) \\
& \mp m^{*} \boldsymbol{r}_{a}^{i} \boldsymbol{\omega}_{+}^{i} \sin \left(\boldsymbol{\omega}_{+}^{i} t\right)-m^{*} \boldsymbol{r}_{s}^{i} \boldsymbol{\omega}_{-}^{i} \sin \left(\boldsymbol{\omega}_{-}^{i} t\right),
\end{aligned}
$$

where the notation $\boldsymbol{r}_{s, a}=\left(\boldsymbol{r}_{1} \pm \boldsymbol{r}_{2}\right) / 2, \boldsymbol{p}_{s, a}=\left(\boldsymbol{p}_{1} \pm \boldsymbol{p}_{2}\right) / 2$ and $\boldsymbol{\omega}_{ \pm}=\left(\sqrt{\omega_{x}^{2} \pm\left(\hat{\Omega}_{12}\right)_{x x}^{2}}, \sqrt{\omega_{y}^{2} \pm\left(\hat{\Omega}_{12}\right)_{y y}^{2}}\right)$ has been introduced. In Eq. (A13), the superscript of a vector denotes the corresponding component of the vector.

Next, the obtained solutions are inserted into Eq. (A3). Finally, after performing the integration over time, one obtains the effective spin-spin coupling for arbitrary orientation of the magnetic field,

$H_{\mathrm{S}-\mathrm{S}}=\sum_{i=x, y} \frac{m^{*} \omega_{i, 12}^{2} E_{\mathrm{Z} 1}^{2}\left(\boldsymbol{l}_{1} \times\left(\boldsymbol{l}_{1} \times \boldsymbol{\gamma}_{i}\right)\right) \cdot \boldsymbol{\sigma}_{1}\left(\boldsymbol{\sigma}_{2} \cdot \boldsymbol{\gamma}_{i}\right)}{4\left(\omega_{x}^{2}-E_{\mathrm{Z} 1}^{2}\right)\left(\omega_{x}^{2}-E_{\mathrm{Z} 2}^{2}\right)}+1 \leftrightarrow 2$,

where $\quad \gamma_{x}=(\beta \cos 2 \gamma,-\alpha-\beta \cos 2 \gamma, 0), \quad \gamma_{y}=$ $(\alpha-\beta \sin 2 \gamma,-\beta \cos 2 \gamma, 0)$, and $\boldsymbol{l}_{i}=\boldsymbol{B}_{i} / B$. For simplicity of notation, $\gamma_{x}$ is referred to as $\gamma$ in the main text.

A few remarks should be made about the result embodied in Eq. (A14). First, from Eq. (A10) we see that $\hat{\Omega}_{12} \propto \boldsymbol{a}_{1} \otimes$ $\boldsymbol{a}_{2}$. Accordingly, the two terms in the sum of Eq. (A14) are proportional to $\cos \alpha_{1} \cos \alpha_{2}$ and $\sin \alpha_{1} \sin \alpha_{2}$; these angles $\alpha_{i}$ are shown in Fig. 8. When only the Rashba SOI is present in the material, the coefficients in front of the two terms are equal, and the coupling is proportional to $\boldsymbol{a}_{1} \cdot \boldsymbol{a}_{2}$. This result gives yet another efficient switching mechanism: when the QDs are rotated in such a way that the two vectors are orthogonal $\left(\boldsymbol{a}_{1} \cdot \boldsymbol{a}_{2}=0\right)$ [42].

\section{APPENDIX B: SPIN-SPIN COUPLING-THE HYBRID SYSTEM}

We start from the Hamiltonian of the system and then apply the SW transformation to remove the first-order SOI term (present only in the single QD). The electrostatic potential $V$ is again expanded around the minimum,

$$
\begin{aligned}
V\left(\boldsymbol{r}_{e}, \boldsymbol{r}_{1}, \boldsymbol{r}_{2}\right)= & V\left(\boldsymbol{r}_{e}, \boldsymbol{r}_{1}\right)+V\left(\boldsymbol{r}_{e}, \boldsymbol{r}_{2}\right) \\
\approx & m^{*} \sum_{i=e, 1,2}\left(\delta \omega_{x}^{2} x_{i}^{2}+\delta \omega_{y}^{2} y_{i}^{2}\right) \\
& +m^{*} \omega_{x, 12}^{2} x_{e}\left(x_{1}+x_{2}\right),
\end{aligned}
$$

where $\boldsymbol{r}_{e}, \boldsymbol{r}_{1}$, and $\boldsymbol{r}_{2}$ are the coordinates with respect to the local minima for the electron in the single QD, and for the two electrons in the DQD, respectively. The terms under the sum only renormalize the frequencies. We do not take them into account; they give only higher-order (in the Coulomb energy) contributions to the final results. The last term acts as an electric field on the DQD. As has been shown in Ref. [21], this term leads to a change in the exchange splitting between the singlet and triplet states in the DQD,

$$
H=H_{0}+H_{\mathrm{Z}}+H_{\mathrm{SO}}+\delta \tilde{J} \tilde{x}_{e} \boldsymbol{S}_{1} \cdot \boldsymbol{S}_{2},
$$

where $\delta \tilde{J}$ is given by

$$
\delta \tilde{J}=\frac{3}{\sinh \left(2 \tilde{l}^{2}\right)} \frac{\omega_{x, 12}^{2}}{\tilde{l} \omega_{\mathrm{D}}^{2}} \epsilon .
$$

$\omega_{\mathrm{D}}$ is the confinement energy in the DQD, and $\tilde{l}$ is the distance between the DQD minima measured in units of a QD size. We assume that the detuning $\epsilon$ is applied to the DQD in order to get the coupling linear in electrostatic coupling.

The SW transformation is given by $S=\left(L_{0}+L_{\mathrm{Z}}+\right.$ $\left.L_{\mathrm{H}}\right)^{-1} H_{\mathrm{SO}}$. Similarly to the previous section, in order to find the inverse Liouvillian, we have to solve the system of ODEs:

$$
\begin{gathered}
\frac{d}{d t} p_{e, x}(t)=-m^{*} \omega_{x}^{2} x_{e}(t)-m^{*} \tilde{J} \boldsymbol{S}_{1} \cdot \boldsymbol{S}_{2}, \\
\frac{d}{d t} p_{e, y}(t)=-m^{*} \omega_{y}^{2} y_{e}(t), \\
\frac{d}{d t} \boldsymbol{r}_{e}(t)=\boldsymbol{p}_{e}(t) / m^{*}
\end{gathered}
$$

The solution is easily obtained: 


$$
p_{e}^{x}(t)=p_{e}^{x} \cos \left(\omega_{x} t\right)-m^{*}\left(x_{e} \omega_{x}+\frac{\tilde{J}}{m^{*} \omega_{x} \lambda} \boldsymbol{S}_{1} \cdot \boldsymbol{S}_{2}\right) \sin \left(\omega_{x} t\right),
$$

$$
p_{e}^{y}(t)=p_{e}^{y} \cos \left(\omega_{y} t\right)-m^{*} y_{e} \omega_{y} \sin \left(\omega_{y} t\right) .
$$

After integration over time, the $S$ transformation is obtained:

$$
\begin{aligned}
-i S= & \sum_{i=x, y} \frac{m^{*} r_{e, i}\left(\mu^{2} g^{2}\left(\boldsymbol{B} \cdot \boldsymbol{\gamma}_{i}\right)(\boldsymbol{B} \cdot \boldsymbol{\sigma})-4 \omega_{i}^{2} \boldsymbol{\gamma}_{i} \cdot \boldsymbol{\sigma}\right)}{8\left(\omega_{i}^{2}-E_{\mathrm{Z}}^{2}\right)} \\
& +\frac{\mu g(\boldsymbol{B} \times \boldsymbol{\sigma}) \cdot \boldsymbol{\gamma}_{i} p_{e, i}}{4\left(\omega_{i}^{2}-E_{\mathrm{Z}}^{2}\right)} \\
& +\frac{\mu^{2} g^{2}\left(\boldsymbol{B} \cdot \boldsymbol{\gamma}_{x}\right)(\boldsymbol{B} \cdot \boldsymbol{\sigma})-4 \omega_{x}^{2} \boldsymbol{\gamma}_{x} \cdot \boldsymbol{\sigma}}{8 \omega_{x}^{2}\left(\omega_{x}^{2}-E_{\mathrm{Z}}^{2}\right) \lambda} \delta \tilde{J} \boldsymbol{S}_{1} \cdot \boldsymbol{S}_{2} .
\end{aligned}
$$

The coupling is contained in the $S, H_{\mathrm{Z}}+\delta \tilde{J} \tilde{x}_{e} S_{1} \cdot S_{2}$ term:

$$
H_{\text {hybrid }}=\frac{3 \mu \mathrm{g} \delta \tilde{J}\left(\boldsymbol{\gamma}_{x} \cdot \boldsymbol{B}\right) \cdot \boldsymbol{\sigma}}{4\left(\omega_{x}^{2}-E_{\mathrm{Z}}^{2}\right) \lambda}\left(\boldsymbol{S}_{1} \cdot \boldsymbol{S}_{2}\right) .
$$

By rewriting the last equation in the pseudospin space, we have obtained the generalization for Eq. (12) for arbitrary magnetic-field orientation.

\section{APPENDIX C: IMPLEMENTATION OF TWO-QUBIT GATES}

Two qubits interacting via the floating gate evolve according to the Hamiltonian $H=H_{\mathrm{S}-\mathrm{S}}+E_{\mathrm{Z}}\left(\sigma_{z}^{1}+\sigma_{z}^{2}\right)$, the sum of the qubit-qubit coupling and the Zeeman term. In general, these contributions do not commute, making it difficult to use the evolution to implement standard entangling gates. However, when the field is perpendicular to the 2DEG substrate, $H_{\mathrm{S}-\mathrm{S}}$ takes the form of Eq. (8), which can be decomposed into two terms as follows:

$$
\begin{aligned}
H_{\mathrm{S}-\mathrm{S}}= & J_{12}\left(\Gamma_{1}-i \Gamma_{2} \sigma_{z}^{1}\right)\left(\sigma_{x}^{1} \sigma_{x}^{2}-\sigma_{y}^{1} \sigma_{y}^{2}\right) / 2 \\
& +J_{12}\left|\boldsymbol{\gamma}_{\mathbf{x}}\right|^{2}\left(\sigma_{x}^{1} \sigma_{x}^{2}+\sigma_{y}^{1} \sigma_{y}^{2}\right) / 2 .
\end{aligned}
$$

Here, $\Gamma_{1}=\left(\left(\boldsymbol{\gamma}_{x}\right)_{x}^{2}-\left(\boldsymbol{\gamma}_{x}\right)_{y}^{2}\right)$ and $\Gamma_{2}=\left(\boldsymbol{\gamma}_{x}\right)_{x}\left(\boldsymbol{\gamma}_{x}\right)_{y}$. The first of these two terms anticommutes with the Zeeman term, whereas the second commutes. That being so, when $E_{\mathrm{Z}} \gg$ $J_{12}\left|\gamma_{\mathbf{X}}\right|^{2}, H_{S-S}$ can be approximated by the second term alone:

$$
\begin{gathered}
H_{\mathrm{S}-\mathrm{S}} \approx H_{\mathrm{S}-\mathrm{S}}^{\prime}=\frac{J_{12}\left|\gamma_{x}\right|^{2}}{2}\left(\sigma_{x}^{1} \sigma_{x}^{2}+\sigma_{y}^{1} \sigma_{y}^{2}\right), \\
H \approx H^{\prime}=H_{\mathrm{S}-\mathrm{S}}^{\prime}+E_{\mathrm{Z}}\left(\sigma_{z}^{1}+\sigma_{z}^{2}\right) / 2 .
\end{gathered}
$$

With this approximation, the coupling and Zeeman terms in $H^{\prime}$ now commute.

We consider the implementation of the gate $\sqrt{\sigma_{x} \sigma_{x}}=$ $\exp \left(-i \sigma_{x}^{1} \sigma_{x}^{2} \pi / 4\right)$, which is locally equivalent to a CNOT.
The Hamiltonian $H^{\prime}$ already contains a $\sigma_{x}^{1} \sigma_{x}^{2}$ term, so implementation of the $\sqrt{\sigma_{x} \sigma_{x}}$ gate requires only that the effects of the other terms be removed by appropriate local rotations. Two possible sequences that can be used to achieve this are

$$
\sqrt{\sigma_{x} \sigma_{x}}=e^{i\left(\sigma_{z}^{1}+\sigma_{z}^{2}\right) E_{\mathrm{Z}} t} e^{-i H^{\prime} t} \sigma_{x}^{1} e^{i\left(\sigma_{z}^{1}+\sigma_{z}^{2}\right) E_{\mathrm{Z}} t} e^{-i H^{\prime} t} \sigma_{x}^{1} \quad \text { and }
$$

$$
\sqrt{\sigma_{x} \sigma_{x}}=\sigma_{x}^{2} e^{-i H^{\prime} t / 2} \sigma_{x}^{1} \sigma_{x}^{2} e^{-i H^{\prime} t / 2} \sigma_{x}^{2} e^{-i H^{\prime} t / 2} \sigma_{x}^{1} \sigma_{x}^{2} e^{-i H^{\prime} t / 2},
$$

where $t=\pi /\left(4 J_{12}|\gamma|^{2}\right)$. The first sequence requires two applications of the qubit-qubit coupling, whereas the second requires four. The main difference is that the former removes the effects of the field through the application of corresponding $z$ rotations after each application of $H^{\prime}$, whereas the latter uses $x$ rotations to negate the sign of the field terms and additional applications of $H^{\prime}$ to cancel them out. The former is therefore simpler to implement, although the latter method will also cancel terms not taken into account in the approximation.

Once the $\sqrt{\sigma_{x} \sigma_{x}}$ has been implemented using either of the above sequences, the CNOT gate, $C$, may be applied using the appropriate local rotations,

$$
C=\sqrt{\sigma_{z}^{1}} \sqrt{\sigma_{x}^{2}} \mathcal{H}^{1} \sqrt{\sigma_{x} \sigma_{x}} \mathcal{H}^{1} .
$$

Here, $\mathcal{H}$ denotes the single-qubit Hadamard rotation.

Since $H^{\prime}$ is an approximation of $H$, the above sequences will yield approximate CNOTs, $C^{\prime}$, when used with the full Hamiltonian. The success of the sequences therefore depends on the fidelity of the gates, $F\left(C^{\prime}\right)$. Ideally this would be defined using a minimization over all possible states of two qubits. However, to characterize the fidelity of an imperfect CNOT, it is sufficient to consider the following four logical states of two qubits: $|+, 0\rangle,|+1\rangle,|-, 0\rangle$, and $|-, 1\rangle$. These are product states which, when acted upon by a perfect CNOT, become the four maximally entangled Bell states $\left|\Phi^{+}\right\rangle,\left|\Psi^{+}\right\rangle,\left|\Phi^{-}\right\rangle$, and $\left|\Psi^{-}\right\rangle$, respectively. As such, the fidelity of an imperfect CNOT may be defined as follows:

$$
F\left(C^{\prime}\right)=\min _{i \in\{+,-\}, j \in\{0,1\}}\left|\left\langle i, j\left|C^{\dagger} C^{\prime}\right| i, j\right\rangle\right|^{2} .
$$

The choice of basis used here ensures that $F\left(C^{\prime}\right)$ gives a good characterization of the properties of $C^{\prime}$ in comparison to a perfect CNOT, especially for the required task of generating entanglement.

In a realistic parameter regime, we can expect that $\left(\boldsymbol{\gamma}_{x}\right)_{x}$ and $\left(\boldsymbol{\gamma}_{x}\right)_{y}$ will be of the same order, and the qubit-qubit coupling will be a few orders of magnitude less than the Zeeman terms. To get a rough idea of what fidelities can be achieved in such cases using the schemes proposed, we average over $10^{4}$ samples for which $\left(\boldsymbol{\gamma}_{x}\right)_{y}$ is randomly assigned values between $\left(\boldsymbol{\gamma}_{x}\right)_{x} / 2$ and $3\left(\boldsymbol{\gamma}_{x}\right)_{x} / 2$ according 
to the uniform distribution, and $J_{12}\left(\gamma_{x}\right)_{x} / E_{\mathrm{Z}}=0.1$. This yields values of $99.33 \%$ and $99.91 \%$ for the sequences of Eqs. (C4) and (C5), respectively. For $J_{12}\left(\gamma_{x}\right)_{x} / E_{\mathrm{Z}}=0.01$, these improve, becoming $99.993 \%$ and $99.998 \%$, respectively.

To compare these values to the thresholds found in schemes for quantum computation, we must first note that imperfect CNOTs in these cases are usually modeled by the perfect implementation of the gate followed by depolarizing noise at a certain probability. It is known that such noisy CNOTs can be used for quantum computation in the surface code if the depolarizing probability is less than $1.1 \%$ [6]. This corresponds to a fidelity, according to the definition above, of $99.17 \%$. The fidelities that may be achieved in the schemes proposed here are well above this value, and, hence, although they do not correspond to the same noise model, we can expect these gates to be equally suitable for fault-tolerant quantum computation.

[1] R. Hanson, L. P. Kouwenhoven, J. R. Petta, S. Tarucha, and L.M.K. Vandersypen, Spins in Few-Electron Quantum Dots, Rev. Mod. Phys. 79, 1217 (2007).

[2] H. Bluhm, S. Foletti, I. Neder, M. Rudner, D. Mahalu, V. Umansky, and A. Yacoby, Dephasing Time of GaAs Electron-Spin Qubits Coupled to a Nuclear Bath Exceeding $200 \mu \mathrm{s}$, Nature Phys. 7, 109 (2010).

[3] A. M. Childs, H. L. Haselgrove, and M. A. Nielsen, Lower Bounds on the Complexity of Simulating Quantum Gates, Phys. Rev. A 68, 052311 (2003).

[4] D. Loss and D. DiVincenzo, Quantum Computation with Quantum Dots, Phys. Rev. A 57, 120 (1998).

[5] R. Raussendorf and J. Harrington, Fault-Tolerant Quantum Computation with High Threshold in Two Dimensions, Phys. Rev. Lett. 98, 190504 (2007).

[6] D.S. Wang, A. G. Fowler, and L.C.L. Hollenberg, Surface Code Quantum Computing with Error Rates over 1\%, Phys. Rev. A 83, 020302 (2011).

[7] A. Wallraff, D. I. Schuster, A. Blais, L. Frunzio, R.-S. Huang, J. Majer, S. Kumar, S. M. Girvin, and R. J. Schoelkopf, Strong Coupling of a Single Photon to a Superconducting Qubit Using Circuit Quantum Electrodynamics, Nature (London) 431, 162 (2004).

[8] L. Childress, A. S. Sørensen, and M. D. Lukin, Mesoscopic Cavity Quantum Electrodynamics with Quantum Dots, Phys. Rev. A 69, 042302 (2004).

[9] M. Trif, V. N. Golovach, and D. Loss, Spin Dynamics in InAs Nanowire Quantum Dots Coupled to a Transmission Line, Phys. Rev. B 77, 045434 (2008).

[10] M. Trif, F. Troiani, D. Stepanenko, and D. Loss, SpinElectric Coupling in Molecular Magnets, Phys. Rev. Lett. 101, 217201 (2008).

[11] I. H. Chan, R. M. Westervelt, K. D. Maranowski, and A. C. Gossard, Strongly Capacitively Coupled Quantum Dots, Appl. Phys. Lett. 80, 1818 (2002); I. H Chan, P Fallahi, R. M Westervelt, K.D Maranowski, and A.C Gossard, Capacitively Coupled Quantum Dots as a SingleElectron Switch, Physica E (Amsterdam) 17, 584 (2003).
[12] F. Kuemmeth, S. Ilani, D. C. Ralph, and P. L. McEuen, Coupling of Spin and Orbital Motion of Electrons in Carbon Nanotubes, Nature (London) 452, 448 (2008).

[13] Y. Hu, H. O. H. Churchill, D. J. Reilly, J. Xiang, C. M. Lieber, and C.M. Marcus, A Ge/Si Heterostructure Nanowire-Based Double Quantum Dot with Integrated Charge Sensor, Nature Nanotech. 2, 622 (2007).

[14] K. Flensberg and C. M. Marcus, Bends in Nanotubes Allow Electric Spin Control and Coupling, Phys. Rev. B 81, 195418 (2010).

[15] J. Levy, Universal Quantum Computation with Spin- $\frac{1}{2}$ Pairs and Heisenberg Exchange, Phys. Rev. Lett. 89, 147902 (2002).

[16] C. Flindt, A. Sørensen, and K. Flensberg, Spin-Orbit Mediated Control of Spin Qubits, Phys. Rev. Lett. 97, 240501 (2006).

[17] M. Trif, V. N. Golovach, and D. Loss, Spin-Spin Coupling in Electrostatically Coupled Quantum Dots, Phys. Rev. B 75, 085307 (2007).

[18] D. Stepanenko and G. Burkard, Quantum Gates Between Capacitively Coupled Double Quantum Dot Two-Spin Qubits, Phys. Rev. B 75, 085324 (2007).

[19] A. C. Johnson, J. R. Petta, J. M. Taylor, A. Yacoby, M. D. Lukin, C. M. Marcus, M. P. Hanson, and A. C. Gossard, Triplet-Singlet Spin Relaxation via Nuclei in a Double Quantum Dot, Nature (London) 435, 925 (2005).

[20] J. Sten, Ellipsoidal Harmonics and Their Application in Electrostatics, J. Electrost. 64, 647 (2006).

[21] G. Burkard, D. Loss, and D. DiVincenzo, Coupled Quantum Dots as Quantum Gates, Phys. Rev. B 59, 2070 (1999).

[22] J.H. Van Vleck, Paramagnetic Relaxation Times for Titanium and Chrome Alum, Phys. Rev. 57, 426 (1940).

[23] V.N. Golovach, A. Khaetskii, and D. Loss, PhononInduced Decay of the Electron Spin in Quantum Dots, Phys. Rev. Lett. 93, 016601 (2004).

[24] We have checked that, for the SOI given by $\alpha \sigma_{x} p_{x}^{3}$, the spin-spin coupling of the form $J_{x x}=$ $-\left(5 \pi^{3} / 2\right) m^{3} \alpha^{2} \alpha_{q}^{3} \alpha_{c}^{3}\left(\partial q_{\text {ind }} / \partial x\right)^{6} \omega_{x}^{6}$ is obtained in the absence of the magnetic field.

[25] It is interesting to note that the derived coupling, Eq. (8), is independent of the orbital states of the QDs and thus insensitive to the fluctuations of a QD electron-charge distribution.

[26] R. Zak, B. Rothlisberger, S. Chesi, and D. Loss, Quantum Computing with Electron Pins in Quantum Dots, Riv. Nuovo Cimento Soc. Ital. Fis. 33, 345 (2010) [http:// paperseek.sif.it/index.php?doi=10.1393/ncr/i2010-10056-y].

[27] D. Stepanenko, M. Rudner, B. I. Halperin, and D. Loss, Singlet-Triplet Splitting in Double Quantum Dots Due to Spin Orbit and Hyperfine Interactions, arXiv:1112.1644.

[28] D.P. DiVincenzo, Fault-Tolerant Architectures for Superconducting Qubits, Phys. Scr. T137, 014020 (2009).

[29] F. H. L. Koppens, C. Buizert, K. J. Tielrooij, I. T. Vink, K. C. Nowack, T. Meunier, L.P. Kouwenhoven, and L. M. K. Vandersypen, Driven Coherent Oscillations of a Single Electron Spin in a Quantum Dot, Nature (London) 442, 766 (2006).

[30] V. N. Golovach, M. Borhani, and D. Loss, Electric-DipoleInduced Spin Resonance in Quantum Dots, Phys. Rev. B 74, 165319 (2006). 
[31] K. C. Nowack, F. H. L. Koppens, Yu. V. Nazarov, and L.M.K Vandersypen, Coherent Control of a Single Electron Spin with Electric Fields, Science 318, 1430 (2007).

[32] S. Nadj-Perge, S. M. Frolov, E. P. A. M. Bakkers, and L. P. Kouwenhoven, Spin-Orbit Qubit in a Semiconductor Nanowire, Nature (London) 468, 1084 (2010).

[33] C. Fasth, A. Fuhrer, L. Samuelson, V. N. Golovach, and D. Loss, Direct Measurement of the Spin-Orbit Interaction in a Two-Electron InAs Nanowire Quantum Dot, Phys. Rev. Lett. 98, 266801 (2007).

[34] C. Klöffel, M. Trif, and D. Loss, Strong Spin-Orbit Interaction and Helical Hole States in Ge/Si Nanowires, Phys. Rev. B 84, 195314 (2011).

[35] D. V. Bulaev, B. Trauzettel, and D. Loss, Spin-Orbit Interaction and Anomalous Spin Relaxation in Carbon Nanotube Quantum Dots, Phys. Rev. B 77, 235301 (2008).

[36] H. O. H. Churchill, A. J. Bestwick, J.W. Harlow, J. Kuemmeth, D. Marcos, C. H. Stwertka, S. K. Watson, and C. M. Marcus, Electronnuclear Interaction in $13 c$
Nanotube Double Quantum Dots, Nature Phys. 5, 321 (2009).

[37] J. Klinovaja, M. J. Schmidt, B. Braunecker, and D. Loss, Helical Modes in Carbon Nanotubes Generated by Strong Electric Fields, Phys. Rev. Lett. 106, 156809 (2011).

[38] J. Fischer and D. Loss, Dealing with Decoherence, Science 324, 1277 (2009).

[39] S. Amasha, K. MacLean, Iuliana P. Radu, D. M. Zumbühl, M. A. Kastner, M.P. Hanson, and A.C. Gossard, Electrical Control of Spin Relaxation in a Quantum Dot, Phys. Rev. Lett. 100, 046803 (2008).

[40] F. Marquardt and V. A. Abalmassov, Spin Relaxation in a Quantum Dot Due to Nyquist Noise, Phys. Rev. B 71, 165325 (2005).

[41] C. Barthel, J. Medford, C. M. Marcus, M. P. Hanson, and A.C. Gossard, Interlaced Dynamical Decoupling and Coherent Operation of a Singlet-Triplet Qubit, Phys. Rev. Lett. 105, 266808 (2010).

[42] Coupling is zero up to the small terms $O\left[\left(\hat{\Omega}_{12}\right)_{x y, y x}^{2} / \mid \omega_{x}^{2}-\right.$ $\left.\omega_{y}^{2} \mid\right]$. 